\title{
A comparative study of GRB-Supernovae
}

\author{
Lekshmi Resmi ${ }^{1}$ and Kuntal Misra ${ }^{2}$ \\ ${ }^{1}$ Dept. of Earth \& Space Sciences, \\ Indian Institute of Space Science \& Technology, \\ Trivandrum-695547, India. \\ email: 1.resmi@iist.ac.in \\ ${ }^{2}$ Aryabhatta Research Institute of Observational Sciences (ARIES) \\ Manora Peak, Nainital-263129, India. \\ email: kuntal@aries.res.in
}

\begin{abstract}
Optical afterglow observations hold the indirect key to type-Ic Supernovae associated with Gamma Ray Bursts. In several cases where there is no spectroscopic confirmation available, presence of the supernova is inferred from the red bump seen in late afterglow light-curves. We do extensive afterglow modeling to extract the supernova contribution as residue. We compare the residual lightcurves of Supernovae associated with GRB041006, GRB030329, GRB050525A and GRB090618.
\end{abstract}

Keywords. gamma rays: bursts, supernovae

\section{Introduction}

Supernovae II/Ibc and Gamma Ray Bursts (GRBs) of the long duration kind are two manifestations of the end of massive stars. Not all massive stars end as long-GRBs. We believe, in certain conditions like if the star has high rotational angular momentum, the end stage will be a Gamma Ray Burst. However, it appears that in many if not all cases of the collapse that leads to a GRB, a type I-c supernova explosion also happens (see Woosley \& Bloom, 2006 for a review). In several low redshift $(z<1)$ GRBs, the supernova start to emerge from the GRB optical afterglow (OA).

Of these relatively low redshift events, only for a handful of them have we been able to obtain a spectroscopic detection of the supernova (Hjorth et al., 2003). The afterglow spectrum is purely non-thermal (believed to be synchrotron origin), with no features. In almost all these cases (except the first ever known association GRB980425/SN1998bw where the OA was not seen) the optical afterglow of the GRB is bright in the beginning, and as afterglow emission gets weaker its blue continuum gives way to the supernova spectrum characterized by a host of spectral lines.

\subsection{SN emission in $O A$ lightcurve}

If the supernova is faint, the spectroscopic identification is not possible, and the presence of the supernova is inferred from the OA lightcurve. The optical afterglow usually decays as a power-law in time as the synchrotron spectrum of the afterglow evolves due to the deceleration of the fireball. If a supernova is present its emission produces a deviation from the power-law decay of the OA. The SN light appears as a 'rebrightening' in the late (of the order of a few days) stages of the afterglow lightcurve (see Fig-1).

Hence the late time optical emission will contain contributions from the optical afterglow, the supernova and the optical emission from the host galaxy of the progenitor star. We use afterglow physical models to subtract the OA and the host galaxy emission. 


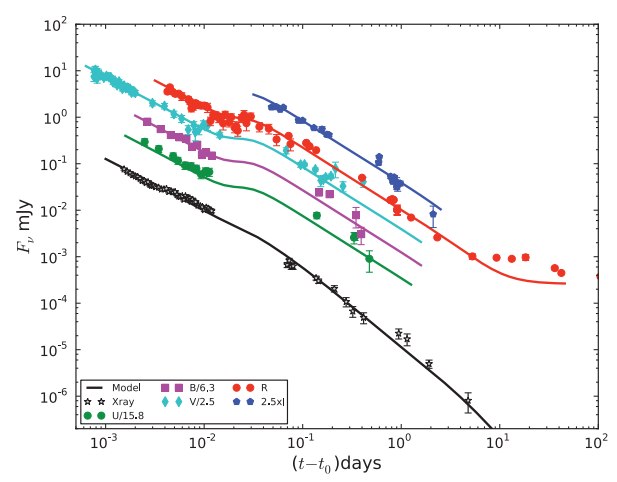

Figure 1. Optical afterglow with the multi-band model that takes care of the afterglow and host-galaxy contribution. In red band we can see the supernova contribution as a rebrightening (Resmi et al. 2012).

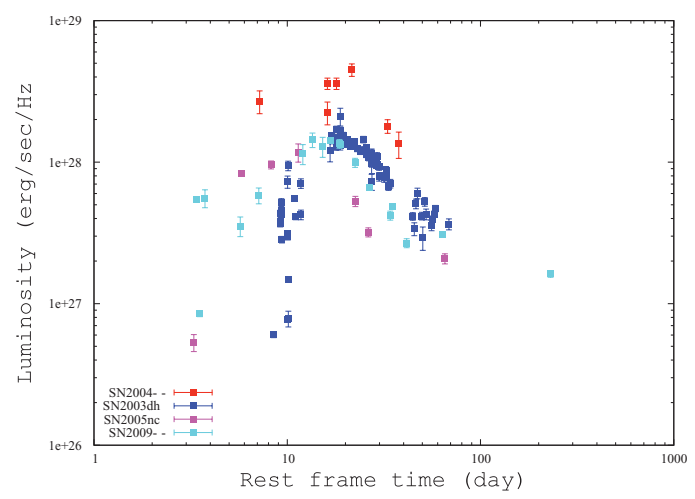

Figure 2. The Supernovae lightcurves in our sample. Luminosity vs. rest-frame time.

\section{Sn lightcurves}

We study four cases where sufficient data from x-ray to radio bands are available for doing a robust afterglow modeling. These are GRB030329/SN2003dh $(\mathrm{z}=0.16)$, GRB041006 $(z=0.71)$, GRB050525A/SN2005nc $(z=0.606)$ and GRB090618 $(z=0.54)$. Of these, SN2003dh was identified spectroscopically too. We assume synchrotron emission from an adiabatically evolving fireball with a given isotropic equivalent energy and initial collimation angle. The flux also depends on the assumed ambient density profile and fractional energy content in non-thermal electrons and magnetic field (see Piran, 1999 for a review). We obtain the SN lightcurves as the residue and calculate the luminosity vs rest-frame time (shown in Fig-2).

Currently our sample contains four sources. A comparative study of the SN lightcurves is underway including more sources in the sample.

\section{References}

Hjorth, J., Sollerman, J., Moller, P., Johan, P. U., et al. 2003, Nature, 423, 847.

Piran, T. 1999, Physics Reports, 314, 575.

Resmi, L., Misra K., Johannesson, G., Castro-Tirado, A. J. et al., 2012 MNRAS, 427, 288.

Woosley, S. E., \& Bloom, J. S., 2006 ARAA, 44, 507. 